\title{
Clinical Approaches to the Near-Death Experiencer
}

\author{
Bruce Greyson, M.D. \\ Barbara Harris, R.T.T. \\ Department of Psychiatry, University of Connecticut \\ Medical School
}

ABSTRACT: The literature on near-death experiences (NDEs) and their aftereffects has focused on the positive personality transformations and spiritual development that often follow an NDE, while it has neglected the emotional and interpersonal problems sometimes precipitated by the experience. We report general guidelines and specific interventions, developed at an interdisciplinary conference, to assist NDErs in coping with psychological difficulties following their experiences.

The growing literature on aftereffects of the NDE has focused on the positive personality transformations that often follow the event (e.g., Bauer, 1985; Flynn, 1982, 1986; Greyson, 1981, 1983; Noyes, 1982-83; Ring, 1984). Despite the fact that about 8 million Americans have had

This report was based on a conference on Clinical Approaches to the Near-Death Experience, sponsored by the International Association for Near-Death Studies, February 5-9, 1984, in Pembroke Pines, FL, and funded in part by a grant from the Institute of Noetic Sciences. The authors gratefully acknowledge the contributions of the other participants in that conference: Patricia Bahr, Boyce Batey, Sharon Batey, Nancy Bush, Kimberly Clark, M.S.W., Diane Corcoran, R.N., Ph.D., Elaine Durham, Linda Fleishman, Charles Flynn, Ph.D., Margaret Huddleston, Joan Kaye, Esther Knecht, Carolyn LewisStone, M.S.W., Jean Marr, Ph.D., John McDonagh, Ph.D., Anne-Marie Meagher, M.D., John Migliaccio, M.Ed., Judith Miller, Ph.D., Raymond Moody, Jr., M.D., Ph.D., Leslee Morabito, Annalee Oaks, R.N., M.A., Ruth Orner, Ph.D., Carol Parrish-Harra, Madelaine Podurgiel, R.N., Ph.D., Laurie Schwartz, Joyce Strom-Paikin, R.N., M.S., Robert Sullivan, Nina Thornburg, M.S.N., Israel Topel, M.D., and Kate Wyatt, Ph.D.

Dr. Greyson is Associate Professor of Psychiatry, and Ms. Harris is Research Assistant, at the University of Connecticut Medical School. Requests for reprints should be sent to Dr. Greyson, Department of Psychiatry, University of Connecticut Health Center, Farm. ington, CT 06032. 
NDEs, according to the 1980-81 Gallup Poll (Gallup, 1982), little is known about the significant intrapsychic and interpersonal difficulties frequently faced by NDErs. Although it is not rare for near-death survivors to feel considerable distress following an NDE that may conflict with their previously held beliefs or attitudes (Atwater, 1983; in press), the popular emphasis on the beneficial consequences of NDEs may inhibit NDErs who experience problems from seeking help.

Occasionally individuals who were totally unprepared to face an experience such as an NDE may doubt their mental stability, but may fear to discuss the NDE with friends or professionals, lest they be rejected, ridiculed, or regarded as psychotic or hysterical. Negative reactions of professionals when the experience is brought up may further alienate the NDEr and inhibit him or her from seeking help in understanding and integrating the experience.

As many NDErs gradually adapt on their own to the experience and its effects, their changing values, attitudes, and interests strain relationships with family and friends who find it difficult to understand or adapt to the NDErs' new beliefs and behavior. On the one hand, some experiencers find themselves ostracized from their families and friends, facing the fears of their significant others that they have come under the influence of evil forces, or that they have somehow become bewitched. On the other hand, media publicity about positive transformations following NDEs may also lead experiencers' families and friends to place the NDEr on a pedestal and to expect unrealistic changes, to expect oracular or healing powers and superhuman patience and forgiveness. They are then disappointed and rejecting when the experiencer is unable to live up to his or her assigned new role as a living saint. Specific intrapsychic problems brought about by the NDE include continuing anger or depression at the return from the near-death state, and difficulty accepting the return, with what some NDErs refer to as "reentry problems" or "withdrawal symptoms"; difficulty reconciling the NDE with prior religious beliefs or with prior values and lifestyles; excessive self-identification with the experience, so that one thinks of oneself first and foremost as an "NDEr"; and the fear that the NDE may reflect mental instability, a concern that often can be surmounted only by redefining normality.

Interpersonal problems brought about by the NDE include a sense of exclusiveness or separation from those who have not had a similar experience; a pervasive fear of ridicule or rejection from others; difficulty in integrating attitude changes with the expectations of family and friends; inability to communicate to others the meaning and impact of the NDE; difficulty in maintaining customary life roles that no longer 
have the same significance after the experience; and accepting the limitations and deficits of human relationships in lieu of unconditional relationships and feelings experienced during the NDE.

Particular problems may face the individual who has had an unpleasant or frightening NDE, or who feels continuing anger or depression at having been revived from the near-death state. Likewise, unique difficulties may face those who have an NDE in childhood or arising out of a suicide attempt.

The response of a counselor, therapist, or health professional to an NDEr may influence critically whether the NDE can be integrated and used as a stimulus for personal growth, or whether it is hidden awaybut not forgotten - as a bizarre event that clashes with the individual's everyday life and may be regarded as a sign of mental instability.

In February 1984, the International Association for Near-Death Studies (IANDS), a worldwide educational and research organization, sponsored a five-day conference in which psychiatrists, psychologists, social workers, nurses, physicians, clergy, sociologists, and NDErs met to share therapeutic strategies, clinical techniques, and insights into the management of problems arising out of the NDE (Anonymous, 1984; Strom-Paikin, 1986). The 32 participants in that conference represented many accumulated years of working with near-death experiencers. The advantage of a panel so diverse in professional training, experience, and theoretical orientation, about one-third of whom had had NDEs themselves, was a breadth of therapeutic strategies and techniques explored and a broadly based consensus as to which approaches were efficacious. However, the panel's heterogeneity precluded any unifying theoretical underpinning to the proceedings, other than an attempt to avoid treating the NDEr as either a diseased patient or as a helpless victim of the experience.

The clinical approaches and interventions outlined below were developed by the IANDS conference participants to help individuals accept the NDE and integrate it more fully into their world view, to decrease their sense of isolation and alienation from significant others who have not shared the experience, to encourage the NDEr to verbalize the insights derived from the experience and thereby to adapt to and grow from the confrontation with death and with an alternate reality, and to reconstruct for themselves a purposeful life in which the NDE and its effects can be balanced with the demands of the individual's everyday existence.

The strategies and techniques below were designed to help NDErs assess the meaning in their lives of the experience and to evolve new concepts of life and death that can validate both the NDE and the life situ- 
ations they return to; and to assist the NDErs' significant others in understanding, accepting, and sharing the experience and its effects to the greatest possible extent. These interventions could ideally be applied on both individual and societal levels in the management of NDEs and their aftereffects, and may lead to the formulation of specific standards of care for NDErs and their significant others. While some of these suggestions are unique to working with near-death survivors, others reflect common sense or therapeutic interventions that would be helpful in any situation. The latter are included below because, while frequently employed, they may be particularly crucial in dealing with NDE-related problems.

\section{Initial Contacts with Near-Death Experiencers}

\section{General Guidelines}

Before any contact with an NDEr, a caregiver should explore his or her own attitudes toward the NDE. One should be well aware of one's own prejudices, both positive and negative, about what the experience means and about people who have them, before interacting with an NDEr.

The caregiver should avoid imposing his or her beliefs or interpretation of the experience on the NDEr. The conversation should be guided by the individual's own account and understanding of his or her NDE, rather than by the caregiver's preconceptions or judgments.

Regardless of what the caregiver believes about the ultimate origin of the experience, the NDE must be respected as an extremely powerful catalyst for transformation. To neglect the profound potential of the experience to initiate both positive and negative changes in personality, beliefs, and physiological function is to discredit what is often the individual's most pressing concern.

One must respect not only the experience but the individual as well. NDEs happen to all types of persons, and the experiencer's rich personal and cultural background, over which the NDE is overlaid, should not be ignored by focusing exclusively on his or her role as an "NDEr."

Labelling either the NDE or the individual with a clinical diagnosis based on the experience is more likely to hinder understanding and alienate the individual than to help. When NDErs do meet diagnostic criteria for a treatable condition, it must be clear to both caregiver and patient that that diagnosis is independent of and unrelated to the NDE 
itself. Attempts to classify the experience as a pathological entity are neither accurate nor helpful.

Honesty is critical in establishing a therapeutic rapport with an NDEr. When appropriate, the caregiver may respectfully share his or her reactions to the experience, without discounting or contradicting the individual's perceptions and attitudes.

The NDEr must be assured of confidentiality; he or she must trust that the caregiver will not divulge the existence or contents of the NDE without permission. Individuals may be cautious about sharing an event as anomalous or as intimate as the NDE until they feel that the interviewer will respect their experience; they may justifiably have concerns about the respect or attitudes of others with whom the interviewer may share that information.

NDErs should be encouraged to express whatever emotions were precipitated by the close brush with death and the NDE. If the NDE itself was characterized by very intense emotion, the individual may still be having unusually intense feelings that need to be shared, vented, or explored.

The caregiver should take a reflective rather than analytical stance; feeding back to the individual his or her descriptions, feelings, and interpretations will help him or her clarify seemingly ineffable experiences, whereas premature interpretations may heighten the NDEr's fear of being misunderstood or ridiculed.

\section{Specific Interventions}

During resuscitation efforts, professional staff should be alert to insensitive comments or behavior. Patients who appear unconscious may be aware of their surroundings, and may later recall behavior that is callous or offensive. When staff say or do things that may be misinterpreted, an explanation to the apparently unconscious patient at the time may prevent having to untangle frightening memories at a later point.

During and immediately after periods of apparent unconsciousness, human contact may be critical in reorienting a patient. Simultaneous verbal and tactile orientation, manually outlining the patient's body boundaries while talking to him or her, may speed the return of bodily consciousness following an NDE.

When interviewing individuals after a close brush with death, caregivers should be alert for clues that he or she has had a NDE. Such individuals may drop subtle hints to test the caregiver's openness to listen, before they risk sharing the experience. 
The most helpful intervention following an NDE is to listen attentively to whatever the individual wants to say. Persons who appear agitated by an experience often feel great pressure to understand it; they usually become more frustrated if they are told not to talk about it, or are sedated into silence. Allowing the NDEr to talk will permit the individual to share, and thereby diffuse, any negative emotions. Unlike a delirious patient, who may become more agitated by verbalizing his or her own confusion, the NDEr will usually be relieved if allowed to struggle until he or she can find the correct words to describe the experience.

The caregiver should not push for details of the experience, but wait for clues that the individual wants to talk further. Persons who have had unusual or intimate NDEs may be reluctant to share the details until they trust the interviewer. One should permit them to describe their experiences at their own pace, watching for subtle hints (tests of one's receptivity) that they want to tell you more.

Each professional must develop his or her own personal ways to encourage talk about the NDE. Using one's own personal style of verbal and nonverbal communicating is the best way to express a willingness to listen openly.

In hospital units or other settings in which close brushes with death are common, it may be helpful to rotate listeners to prevent burnout. NDErs excited about their extraordinary experience may need periodic fresh listeners who have the time and patience to hear them out.

Caregivers should validate the individual's subjective experience in and of itself. However the individual or the caregiver interprets the NDE and its meaning, the experience itself must be recognized as subjectively real and powerful, and cannot be dismissed as trivial or inconsequential.

The caregiver should be sensitive to the individual's interpretation of the NDE. One should listen for clues as to how he or she understands or makes sense of the experience, and, if necessary, help the individual clarify that interpretation by reflecting his or her own words, rather than by imposing one's own opinion of the experience.

One of the most helpful interventions is education about NDEs in general. Straightforward factual information shared in a nonjudgmental way often alleviates concern about the implications or consequences of an NDE to an individual.

Individuals should be advised of the universality of NDEs, while granted the uniqueness of their particular experiences. While individuals are usually relieved to learn how common NDEs are, caregivers must guard against using the NDE's commonness to trivialize any individual's experience or its unique impact on his or her life.

When individuals appear distressed following an NDE, they should 
be helped to identify the specific problems related to the experience. Caregivers should explore possible intrapsychic and interpersonal difficulties, utilizing the individual's knowledge of and fantasies about his or her individual situation and personality.

Caregivers should tailor their interventions to the specific problems needing attention. No two individuals will have the same NDE, the same previous personality, or the same life situation to which to return.

Finally, individuals may need guidance in arranging necessary medical or psychosocial care. For concerns centered around their NDEs, they should be referred to appropriate resources, including other NDErs or local knowledgeable professionals, literature on near-death studies, the national IANDS office at the University of Connecticut, or local Friends of IANDS support groups that exist in many cities.

\section{Continuing Contacts with Near-Death Experiencers}

\section{General Guidelines}

The caregiver who attempts to work with an NDEr past the initial contacts should realize that the NDE raises issues about existence and purpose not necessarily addressed in other therapeutic relationships. The profound aftereffects of an NDE often influence the therapist's psychospiritual growth as well as the NDEr's. Caregivers should decide whether they wish to accept that challenge before entering into an ongoing relationship focused on an NDE.

Having decided to continue working with an NDEr, a therapist should clarify his or her own expectations, and those of the NDEr. The caregiver must ensure that he or she understands what help the NDEr wants from the relationship, and what outcomes the NDEr desires, and furthermore ensure that the NDEr understands the therapist's expectations of the relationship and its outcomes. It should not be assumed that therapy begun prior to an NDE will continue along the same course after the experience; even though the individual's underlying problems and personality may be unchanged, his or her life goals and priorities may be dramatically altered by the NDE.

Areas to be addressed in the relationship may need to be limited. Taking into consideration the individual's personality and level of functioning prior to the NDE, one must clarify what problems result from the NDE as opposed to some other source. Attempting to help an individual with NDE-related problems and also to counsel regarding other problems, or to treat any coexisting psychopathology, may lead to conflict- 
ing interests or objectives. For example, some longstanding psychopathology may be alleviated by helping the patient adapt to societal norms, while the same individual's NDE-related distress may be increased by attempting to adapt to values no longer seen as meaningful. Caregivers working with an NDE may need to refer that individual's problems that are not related to the NDE to another therapist.

Regarding the NDE as a pathologic symptom is countertherapeutic. The NDE by itself is neither a problem nor an indication of other problems.

Counselors should be flexible in regard to length and frequency of sessions. Since the NDE may differ so radically from the individual's other experiences and be very difficult to describe in words, its exploration may take unusually long sessions, and may unmask overwhelming emotions and thought patterns that require frequent sessions.

Both participants in the therapeutic relationship must continually work towards mutual trust. Because of the striking contrast between mundane reality and that of the NDE, it may take longer than usual for an NDEr to trust even the most sensitive caregiver with some parts of the experience and its aftereffects. The extramundane reality of the NDE may also make it difficult for even the most openminded therapist to trust some of the NDEr's memories or interpretations of the experience.

The therapist should avoid glorifying or idealizing the NDE and its aftereffects. The newness and uniqueness of the experience may lead both the NDEr and the caregiver to regard it, or the NDEr, in unrealistically romanticized terms. Likewise, remarkable physical, emotional, or mental aftereffects may be endowed with undue importance simply because they are so different from the individual's prior functioning. Apparent paranormal effects are particularly liable to capture the interest of both therapist and NDEr, potentially interfering with a fuller understanding of the experience and its meaning for the individual's growth.

Caregivers should be alert for what Carl Jung (1969) called "inflation": if the individual overidentifies with the uniqueness of the NDE and subsequent changes, he or she may develop a distorted perspective of the experience within the context of his or her entire being. Jung cautioned (1969, p. 85): "The danger of wanting to understand the meaning is overvaluation of the content." Identification with an elite cohort of NDErs may lead to alienation from others who have not had similar experiences, and to an undue sense of superiority and consequent neglect of basic problems.

Regarding the NDEr as a victim of his or her experience is counter- 
therapeutic. Conversely, helping the individual to appreciate his or her active role in the creation or unfolding of the NDE may help the individual understand and resolve problems arising out of the experience.

Rigid adherence to traditional professional roles may undermine a caregiver's therapeutic rapport with an NDEr. Since manmade distinctions and labels may lose their meaning after an NDE, objective or analytic distancing between partners in the therapeutic relationship, or diagnostic labelling of the NDEr's problems, may interfere with, rather than foster, fuller understanding of his or her difficulties.

The caregiver should avoid an appearance of abandoning the NDEr. Frustration is common in trying to communicate NDEs and their effects, and individuals may give up trying if they perceive caregivers as giving up. Furthermore, individuals who feel they were "sent back" to life against their will may feel rejected in their wish to remain in the extramundane reality of the NDE, and may be particularly sensitive to further rejection.

\section{Specific Interventions}

The NDE itself should be distinguished clearly from its aftereffects. Individuals must feel free to reject, resist, or moderate unwanted aftereffects without having to devalue the NDE itself. While the NDE and its recollection are permanent facts in the individual's subsequent life, various aftereffects can wax and wane in a natural course or be moderated by psychotherapy.

The individual's fears and unwanted aftereffects following an NDE should also be explored. Whether or not an NDEr's fears regarding the experience's consequences are realistic, both interpersonal and physical aftereffects may be related concretely to continuing problems.

The individual must learn to balance his or her perspective toward the more striking aftereffects within the context of the entire NDE. Apparent paranormal or other unusual effects may capture so much interest by their novelty that the NDEr may neglect other important aspects of the experience or other aftereffects that may be more critical in fostering psychospiritual growth.

Likewise, the individual must learn to balance his or her perspective toward the NDE within the context of his or her entire life. The NDE and its aftereffects certainly cannot be ignored, but neither should they be focused on to the neglect of other aspects of the individual's life. In that context, the therapist may help individuals who are "addicted" to 
the NDE or its aftereffects to withdraw gradually or to moderate their focus on the experience.

In withdrawing an individual from the awesome reality of the NDE, it may help to point out that problems may be insoluble on the level that generated them. NDErs often report that certain problems generated in daily interactions needed the insights afforded by the NDE to solve them. So too certain problems generated in the NDE may be resolvable only in the arena of daily existence, and not by reverting to the alternate reality of the NDE.

The therapist should explore prominent features of the NDE for clues regarding sources of continuing problems after the experience. Whatever components of the NDE, such as the life review, precognitive visions, or certain strong feelings, appeared most prominent in the experience itself or in its recollection may yield critical insights into the meaning of continuing problems. Investigation of those particular features may help elucidate those problems.

Caregivers should also explore an individual's sense of having a concrete purpose or mission after surviving an NDE. If he or she identifies a specific reason for having been "sent back" to life, that "unfinished business" may be related critically to continuing problems.

Caregivers should also investigate the NDEr's sense of "divine manipulation." A sense of being used, either positively or negatively, for a higher purpose, may contribute to ongoing problems following an NDE.

With individuals who report having chosen to return to life, or having chosen not to return, that decision should be explored. Continuing problems may be related to regrets, guilt, or ambivalence over having returned, or conversely over having wished not to return.

The therapist should encourage grief work for those parts of the ego that may have died. Even unwanted parts of the ego that were abandoned or transcended in the NDE may need to be grieved for.

Therapists should particularly pursue anomalous details of the NDE and the individual's free associations to those details. Encouraging the individual to interpret NDE imagery on multiple levels, as one might interpret dream imagery, may yield valuable insights into subsequent difficulties.

Techniques for inducing altered states of consciousness may aid in recall of further details of the NDE, and may help train the NDEr to shift voluntarily between states of consciousness. Techniques for integrating the right and left hemispheres in particular may facilitate the individual's ability to use practically the insights gained in the NDE. Im- 
agery and projective techniques may prove invaluable in uncovering and expressing changes that may be difficult to describe verbally.

The therapist should also elucidate family dynamics as they relate to the NDE. Subtle changes in family interactions as a result of NDEinduced changes in values or attitudes may contribute to ongoing problems. Home visits may be useful in assessing interactions with significant others, and the reactions of family and friends to the NDEr and to hearing about the NDE. The caregiver should offer family therapy, or refer to a family therapist, if family dynamics have been disrupted sufficiently.

Psychosocial rehabilitation may help the individual learn to adapt to daily demands that may no longer seem relevant to him or her but still are necessary. For example, the timeless quality of the NDE may make it difficult for individuals subsequently to remain grounded in the present. Some persons may become preoccupied with the past after a profound life review, while others may fixate on the future as a result of profound precognitive visions in the NDE. In extreme cases, a firm here-and-now therapeutic focus may be necessary to permit the NDEr to function effectively in the present.

The NDEr's external reality may have to be altered to reflect his or her internal changes. If the individual's new attitudes, beliefs, or values are irreconcilable with his or her old roles or environment, then those may need to be altered to conform to the NDEr's new goals and priorities. In extreme cases, counseling may need to address the dissolution of careers or major relationships.

Finally, the caregiver's ultimate utility to the NDEr may be in helping to channel those insights and values gained during the experience into constructive action. Those altered attitudes, beliefs, and life goals that can create conflict in the individual's environment can also be instrumental in altering that environment for the better. The best way for the NDEr to validate and reconcile internally the experience and its effects is to use what he or she has learned to help others. The therapeutic work is complete when the individual has found a way to actualize in daily life the love he or she received in the NDE.

\section{References}

Anonymous. Workshop outcomes to benefit clinicians, experiencers. (1984). Vital Signs, $3,(4), 3-4$.

Atwater, P. M. H. (1983). Coming back: the after-effects of survival. Vital Signs, 3, (2), 16. Atwater, P. M. H. (In press). Coming Back. New York, NY: Dodd, Mead. 
Bauer, M. (1985). Near-death experiences and attitude change. Anabiosis, 5, 39-47. Flynn, C. P. (1986). After the Beyond: Transformation and the Near-Death Experience. New York, NY: Prentice-Hall.

Flynn, C. P. (1982). Meanings and implications of NDEr transformations: some preliminary findings and implications. Anabiosis, 2, 3-14.

Gallup G., Jr. (1982). Adventures in Immortality: A Look Beyond the Threshold of Death. New York, NY: McGraw-Hill.

Greyson, B. (1981). Near-death experiences and attempted suicide. Suicide and LifeThreatening Behavior. 11, 10-16.

Greyson, B. (1983). Near-death experiences and personal values. American Journal of Psychiatry, 140, 618-620.

Jung, C. G. (1969). The transcendent function. In R. F .C. Hull (Trans.), The Structure and Dynamics of the Psyche, 2nd Ed. Princeton, NJ: Princeton University Press.

Noyes, R., Jr. (1982-83). The human experience with death or, what can we learn from near-death experiences? Omega, 13, 251-259.

Ring, K. (1984). Heading Toward Omega: In Search of the Meaning of the Near-Death Experience. New York, NY: William Morrow.

Strom-Paikin, J. (1986). Studying the NDE phenomenon. American Journal of Nursing. $86,420-421$. 